\section{Entrevista al doctor Hugo Caballero Cornejo, maestro de generaciones, investigador, referente a nivel nacional e internacional en la odontología forense "especialidad del milenio"}

\author{
Interview to doctor Hugo Caballero \\ Cornejo, teacher of generations, \\ researcher, the reference to national and \\ international level in forensic dentistry \\ "specialty of the millennium"
}

\section{Entrevista}

Daniel Guillermo Suárez Ponce ${ }^{1, a}$

1 Universidad Nacional Mayor de San Marcos,

Facultad de Odontología. Lima, Perú.

a Doctor en Estomatología.

\section{Correspondencia:}

Daniel Guillermo Suárez Ponce

Correo electrónico: dsuarezp@unmsm.edu.pe

Calle Germán Amézaga №375. Ciudad Uni-

versitaria. Lima 1. Perú.

Conflicto de intereses: Los autores declaran no tener conflictos de interés.

Fuente de financiamiento: Autofinanciado

Fecha de recepción: 25/06/18

Fecha de aceptación: 06/08/18

\title{
Resumen
}

En la entrevista realizada al profesor Hugo Caballero Cornejo conoceremos acerca de sus inicios, logros, su aporte académico, científico y de investigación a la odontología forense, siendo un profesional de reconocido prestigio a nivel nacional e internacional. Se conversó sobre el pasado, presente y futuro de la profesión en la especialidad que se desenvuelve a través de sus diversas áreas temáticas, tales como la responsabilidad profesional y la identificación humana, estableciendo que la especialidad de la "odontología forense es la especialidad del milenio" por ser la rectora de todas las especialidades de la profesión, por las implicancias legales y éticas que el odontólogo debe cumplir en el ejercicio profesional. El connotado docente es autor y editor del libro "Odontología Legal y Forense".

Palabras clave: Odontología; Odontología forense; Responsabilidad legal; Profesor universitario.

\begin{abstract}
In the interview with the professor Hugo Caballero Cornejo, we will learn about his beginnings, his achievements, his academic, scientific and research contribution to forensic dentistry, being a professional of recognized prestige at a national and international level. We talked about the past, the present and the future of the profession in the specialty that is developed through its various thematic areas, such as professional responsibility and human identification, establishing that the specialty of "forensic dentistry is the specialty of the millennium" for being the rector of all the specialties of the profession, for legal and ethical implications that the dentist must obey in professional practice. The notorious teacher is the author and editor of the book "Legal and Forensic Dentistry".
\end{abstract}

Keywords: Dentistry; Forensic dentistry; Liability, legal; University professor.

(C) Los autores. Este artículo es publicado por la revista Odontología Sanmarquina de la Facultad de Odontología, Universidad Nacional Mayor de San Marcos. Este es un artículo de acceso abierto, distribuido bajo los términos de la licencia Creative Commons Atribucion - No Comercia_Compartir Igual 4.0 Internacional. (http://creativecommons.org/licenses/by-nc-sa/4.0/) que permite el uso no comercial, distribución y reproducción en cualquier medio, siempre que la obra original sea debidamente citada. 


\section{Introducción}

Hugo Caballero Cornejo (Huancayo-Perú, 1941) es cirujano dentista sanmarquino, especialista en Odontología Forense, maestro en Investigación y Docencia Universitaria, doctor en Educación y docente investigador. Se ha desempeñado como Coronel (S), Perito Odontólogo y Jefe del Departamento Odontográfico de la Policía Nacional del Perú.

Es miembro fundador del Colegio Odontológico del Perú, de la Sociedad Peruana de Medicina Legal, de la Asociación Sanmarquina de Odontología Legal y Forense, además, es miembro activo de la Asociación de Odontólogos Forenses de Sudamérica, así como, miembro honorario de la Sociedad Argentina de Odontología Legal, del Instituto de Integración Latinoamericana (Mendoza - Argentina) y del Instituto de Odontología y Ciencias Clínicas.

Actualmente es profesor de las asignaturas de Ética y Deontología, Estomatología Legal y Forense, es coordinador y profesor de la segunda especialidad profesional en Odontología Forense de la Universidad Nacional Mayor de San Marcos. Es conferencista y ha recibido reconocimientos por su destacada labor profesional a nivel nacional e internacional. Es autor y editor del Libro "Odontología Legal y Forense".

\section{Entrevista}

\section{¿Cuál es el campo de acción de la odontología forense?}

En primer lugar, hay que aclarar que en nuestro país se conoce a la especialidad como odontología forense. Autores de otros países y el suscrito dividimos a la especialidad en dos: la odontología legal y la odontología forense. La odontología legal, está referida al conocimiento de las distintas normas legales que se ocupan del que hacer de nuestra profesión, para el mejor y correcto ejercicio profesional desde el punto de vista legal y ético. La odontología forense, es la aplicación de los conocimientos odontológicos con la finalidad de resolver determinados problemas que se plantean al Derecho, para ayudar a resolver problemas jurídicos.

\section{¿Cuál ha sido la historia de la odontología forense?}

El 27 de noviembre de 1962, se produce en nuestro país la caída del avión de la compañía VARIG, los odontólogos de la Sanidad de la Policía de ese tiempo, identificaron la mayoría de las víctimas. Luego, se crea la Sección de Odontología Forense en la Policía de Investigaciones del Perú el 15 de junio de 1963, perteneciendo a la División de Identificación integrante de la Dirección de Criminalística; teniendo como uno de sus integrantes pioneros y creadores a mi maestro el Dr. Edgard Fernández Chirinos, el suscrito ingresa a la institución el año 1967. Como puede apreciarse estamos próximos a cumplir 55 años de existencia de la odontología forense en nuestro país.
¿En qué instituciones estatales o privadas se puede ejercer la
odontología forense?

Las instituciones estatales en las que se puede laborar son el Ministerio Público (a nivel nacional), la Policía Nacional del Perú, universidades nacionales (docencia), Ministerio de Salud (auditoría - asesoramiento), Superintendencia Nacional de Salud: SUSALUD (auditoría - asesoría de derechos del paciente), Ministerio de Trabajo (salud ocupacional, Superintendencia Nacional de Fiscalización Laboral: SUNAFIL), Ministerio de Justicia (peritos judiciales), Seguro Social de Salud: EsSALUD (auditoría - asesoramiento). Las instituciones privadas en las que se puede laborar son las compañías de seguros (peritos odontólogos - asesoramiento), clínicas (auditoría - salud ocupacional - asesoramiento), universidades privadas (docencia). Además, los peritos odontólogos pueden dar asesoramientos, actuar como peritos de parte o dedicarse a la salud ocupacional en forma particular. Como se puede apreciar el campo es muy amplio.

La odontología forense es la especialidad del milenio ¿Qué consideraciones ha tenido al respecto?

He manifestado que la odontología forense es la especialidad del milenio basado en que la especialidad es la rectora de todas las especialidades de la profesión por las normas legales y éticas que el odontólogo tiene que cumplir en el ejercicio profesional. El problema es la falta de especialistas que trasmitan bien el contenido de la asignatura en las diferentes universidades del país, no hay mucho conocimiento de la especialidad. Los profesionales al ejercer la profesión solo creen que el aspecto cognitivo es el que impera en los tratamientos en todas las especialidades de la carrera, sin darse cuenta que aparte de ello se necesita también conocer bien las normas legales y éticas para ejercer correctamente la profesión, justamente es lo que hace la odontología forense, respetar los derechos de los pacientes, ello evitaría muchos casos de mala praxis, denuncias de los pacientes, llegando inclusive a casos muy graves de faltas e incluso delitos. Para eso está la odontología forense como rectora y normativa de todas las especialidades de la odontología.

¿Cuál es el concepto o definición de responsabilidad profesional?

La definición es clara y dice: es la obligación que tienen los distintos profesionales, y entre ellos el odontólogo; de reparar, responder y resarcir los daños que haya generado a los pacientes como consecuencia de faltas cometidas en el ejercicio de su profesión. Está normado por el Código Civil, Código Penal, Código de Ética del Colegio Odontológico del Perú, Ley General de Salud, etc. Tiene una connotación civil, penal, jurídica, ética, administrativa y laboral. Si el Odontólogo no conoce bien el concepto ¿cómo puede ejercer con responsabilidad profesional? Pero, generalmente sucede así. He tenido oportunidad de auscultar en mis alumnos de pregrado, posgrado, participantes en cursos, congresos nacionales, 
y otros que, tanto estudiantes y profesionales, no tienen claro el concepto de responsabilidad profesional, es por eso que cometen una serie de faltas, delitos y son denunciados.

\section{¿Qué debería conocer bien el odontólogo con respecto al tema?}

En primer lugar; conocer, interpretar y aplicar bien el concepto de responsabilidad profesional, para que cuando cometa una falta de tipo culposa (impericia, imprudencia, negligencia) en el ejercicio de su profesión, su comportamiento con el paciente sea de acuerdo con el concepto de responsabilidad profesional. Podría decirse que es un protocolo que tiene que seguir para su desenvolvimiento con el paciente cuando suceden estos casos. Por eso, siempre digo a mis alumnos, cuando se trata de responsabilidad profesional, deben conocer y cumplir con la norma legal y con el aspecto ético para ejercer mejor su profesión, de esta manera, el paciente no será vulnerado en sus derechos que están normados en varios dispositivos legales.

¿Qué precauciones debe tener el cirujano dentista u odontólogo especialista para no verse involucrado en un caso de responsabilidad profesional?

Lo que podría recomendarle es que cumpla con el tratamiento que realiza a su paciente. En el aspecto cognitivo prepararse bien como profesional en sus conocimientos, en la destreza que debe tener; luego cumplir con los dispositivos legales que norman los derechos de los pacientes y que regulan el ejercicio profesional, sin descuidar el aspecto ético que es importante, además, comportarse según los principios morales que la sociedad le indica, así como aplicando todos los valores que le fueron inculcados por los padres en un inicio y luego por la sociedad en que se desenvuelve. En caso tengan un problema de culpa en el ejercicio de la profesión, a pesar de las precauciones que pueda tomar, no abandonar al paciente, tratar de solucionar correctamente lo sucedido de acuerdo con la ley, antes que lleguen a denunciarlo. El paciente realiza la denuncia no por la culpa cometi$\mathrm{da}$, sino porque se abandona al paciente con una serie de evasivas de parte del profesional para no asumir su responsabilidad.

En caso un cirujano dentista u odontólogo especialista esté envuelto en un caso de responsabilidad profesional ¿Qué acciones debe tomar para afrontar el caso?

Si no ha logrado solucionar el problema suscitado con el paciente, tratando de reparar y solucionar la falta culposa que se haya podido cometer, entonces debería seguir las siguientes acciones: en primer lugar, asesorarse con especialistas en Odontología Forense, para que lo orienten en la solución de la falta cometida y evitar ser denunciado. Si el profesional ha sido denunciado, tratar de llegar a la conciliación con su paciente. En caso fuera denunciado en el Poder Judicial o Ministerio Público, contactarse con un abogado entendido en denuncias de responsabilidad profesional para que asuma su defensa.
Tenemos conocimiento que el número de denuncias por responsabilidad profesional ha aumentado en las facultades de odontología de la capital y al interior del país ¿Qué acciones deberian realizar las autoridades para prevenir estas denuncias y/o enfrentarlas en caso se presenten?

Primero, las autoridades de la facultad deben asesorarse bien con los odontólogos forenses que se encuentran en calidad de docentes de la especialidad en la facultad., Estos especialistas; con amplios conocimientos en responsabilidad profesional, en peritajes odontológicos y médicos, en auditoria odontológica deberían dar las pautas de cómo debe ser el comportamiento profesional en base a los dispositivos legales y éticos que regulan el ejercicio profesional. Dentro de acciones concretas está la creación de un Comité de Ética y Conciliación para que en caso se presente una denuncia por parte de un paciente por mala praxis u otros motivos se trate de conciliar el problema de forma justa y sin espíritu de cuerpo para evitar que el paciente recurra a otras instancias.

En el caso de nuestra facultad, es importante hacer de conocimiento a los estudiantes - de pregrado y posgrado - y docentes de todos los departamentos el Código de Ética Profesional y Deontología del Colegio Odontológico del Perú, Código de Ética de los alumnos, Código de Ética de las Segundas Especialidades, Código de Ética del Personal que labora en la facultad, Reglamento para los alumnos que se encuentran en las Clínicas emanados por SUSALUD, SUNAFIL; todo lo manifestado ya se sugirió a las autoridades después de una supervisión interna con especialistas de odontología forense que se realizó en la facultad el ańo pasado y fue expuesto en el Consejo de Facultad.

Conocemos su participación en casos mediáticos en los que su participación como odontólogo forense ha llevado a la identificación de cadáveres o restos humanos esqueletizados involucrados en estos casos. En este sentido ¿Cuál es el concepto de identificación? ¿Cómo es posible identificar a un cadáver o restos esqueletizados a través de la odontología forense?

Identificación, como lo digo en mi libro Odontología Legal y Forense, al igual que otros autores, es un procedimiento técnico científico por el cual se precisa de manera indubitable, la personalidad de un individuo. Al final de todo el proceso de identificación se logra determinar la personalidad de un individuo, lógicamente, se tiene que demostrar primero que la(s) muestra(s) (cadáveres, restos esqueletizados u otros similares) que se está homologando con los datos premortem sean idénticos (dícese de lo que en circunstancias o accidentes es la misma cosa con la cual se compara). Si se logra demostrar que son idénticos, se llegaría a la identidad del cadáver, los restos humanos esqueletizados $\mathrm{u}$ otros restos humanos. En conclusión, la identificación viene a ser un conjunto de características que distinguen a una persona de las demás, por lo tanto, hablamos de una identificación plena. Se tiene una serie de técnicas y métodos basados en la alta resistencia que tienen las piezas dentarias (en base a 
su constitución prevalente inorgánica) al tiempo, fuego y humedad. Existen muchos métodos y técnicas odontológicas, como la odontometría, huellas de mordedura, rugopalatinoscopía, odontograma de identificación, radiología bucal, fotografía bucal, además, la estimación de la especie, sexo, edad, grupo racial y estatura coadyuvan al proceso de identificación humana.

Muchos creen que la labor del odontólogo forense durante la identificación es la comparación de odontograma antemortem con un odontograma posmortem ¿Cómo se identifica a un cadáver o restos humanos esqueletizados sino se cuenta con el odontograma antemortem?

Para empezar tengo un principio que siempre lo he expresado tanto a nivel nacional como internacional, que identificación es comparación. Si no se tiene información premortem y sólo del posmortem no se tendría como homologar. Si no se cuenta con la información antemortem se debe tratar de entrevistar a la familia para tener información de carácter odontológico de la víctima y poder emplear otras técnicas, que si bien es cierto, no se lograría una identificación positiva se puede llegar a una identificación probable, la búsqueda de información es una labor ardua, pero es necesaria hacerla.

¿Recuerda usted algún(os) caso(s) mediático(s) o trascendente(s) en los que se haya puesto a prueba su experiencia como perito odontológico forense?

En mis 31 años de servicios reales y efectivos en la Policía Nacional del Perú (ingresé a la gloriosa Policía de Investigaciones del Perú, donde inicié mi formación como perito), tuve oportunidad de participar en numerosos casos de identificación, siendo los más trascendentes los desastres aéreos, por ejemplo, la compañía Faucett en el Cuzco, dos de la compañía LANSA, Aerocóndor. Los más emblemáticos fueron el avión Fokker (relacionado al equipo de fútbol de Alianza Lima), otros casos como "El sexto", el caso Jessica y el último, la identificación de los estudiantes de "La Cantuta" que fue de mucha relevancia e importancia a nivel nacional e internacional, donde todo el equipo de peritos cumplió una labor encomiable y definitiva para el esclarecimiento del hecho.

El avance de la tecnología en los procedimientos de identificación a través del ADN avanza a pasos acelerados ¿Cree usted que en algún momento se pueda prescindir del odontólogo forense para los casos de identificación?

Igual preocupación y pregunta me hicieron los odontólogos forenses de la Policía Nacional del Perú cuando me encargaron crear el Departamento de $\mathrm{ADN}$ en la Dirección de Criminalística de la Policía Nacional del Perú como consecuencia de los hechos acaecidos con los estudiantes de La Cantuta, el día 12 de julio de 1992, debido a las dificultades para lograr la identificación. Se pensó que el $\mathrm{ADN}$ iba a reemplazar a todos los métodos de identificación, pero no es así; la Organización Internacional de Policía Criminal (INTERPOL) manifiesta en su Manual para Identificación de Víctimas en Grandes Catástrofes que los únicos métodos indubitables de identificación son la dactiloscopía, el sistema odontológico (odontograma y radiología bucal) y el ADN. En mi experiencia, el ADN tiene muchas implicancias: el costo elevado de los insumos, el tiempo que lleva el proceso que es propio de la técnica del ADN, la muestra, cuya condición debe ser buena para obtener el ADN del cadáver o resto esqueletizado, pero sobre todo, en ocasiones ni el ADN puede establecer la identidad. No debe olvidarse que la identificación es comparación y si no hay una muestra de homologación, ninguno de los tres métodos que dice la INTERPOL sería eficaz. Sigo siendo participe que el mejor método es el odontograma de identificación por una serie de ventajas que tiene y por haberlo usado en toda mi trayectoria como odontólogo forense en forma prioritaria.

Tenemos conocimiento de que existen otros medios de identificación como la rugoscopia y la queiloscopia ¿Ha desarrollado usted algún método de identificación que pueda revolucionar a la odontología y antropología fForense en el proceso de identificación de una persona sin identidad?

La rugoscopía y la queiloscopía no son métodos fehacientes de identificación, la INTERPOL no los toma en cuenta, ambos métodos se pueden considerar siempre que haya una base de datos premortem; así como en casos especiales, por ejemplo, los desdentados totales en los que no hay piezas dentarias se podría utilizar las rugas palatinas. Respecto a la queiloscopía se debería tener también una base de datos premortem para poder homologarlos con la información posmortem, siempre y cuando estén intactas, aprovechables y se pueda tomar la muestra. Es importante hacer una aclaración, la queiloscopía es una técnica que se usa más para identificar al autor de un delito que a la víctima.

He desarrollado, actualizado y perfeccionado el odontograma de identificación y me han solicitado para su estudio, análisis y posiblemente su implementación en países como Argentina, Ecuador y Puerto Rico. En mi opinión y experiencia, a través de los 51 años que tengo como odontólogo forense, sigo insistiendo que el mejor método que tenemos para identificar a personas vivas o cadáveres es el odontograma de identificación, tal es así, que en el Congreso de la Republica, se ha elaborado un Proyecto de Ley para crear el Registro Nacional de Identificación Odontológica-RENIOD, la propuesta fue realizada por el Congresista General Marco Enrique Miyashiro Arashiro.

Existen varias investigaciones cientificas relacionadas a la estimación de la edad ¿Qué utilidad o impacto tienen dichas investigaciones en la sociedad y en especial en el espectro de acción de la odontología forense?

En la especialidad hay muchos temas para investigar, se tiene un campo de acción muy amplio, se investiga no solo en la estimación de la edad, hay temas para investigar como la determinación del sexo, estatura, especie, utilizando mediciones de las piezas dentarias. De igual manera, la estimación del grupo racial a través de sus características, mediciones del cráneo y por medio de las piezas dentarias; asimismo, la estimación de la edad desde que son bebes, en la infancia, pubertad, adolescencia, adultos y en la etapa senil, todos tienen sus métodos y 
técnicas. La utilidad que tiene la estimación de la edad es importante y coadyuva en el proceso para lograr la identidad. En nińos y niñas en estado de abandono, personas extraviadas, personas en tránsito a la mayoría de edad involucradas en hechos delictuosos, en la identificación de restos óseos humanos que guardan relación con graves violaciones a los derechos humanos y en todo tipo de identificación humana.

Considerando que la identificación es una de las principales actividades del odontólogo forense ¿Por qué el número de investigaciones cientificas en torno a la identificación es menor respecto a las que se basan en la predicción de la edad?

Sí hay diversas investigaciones basadas en otros temas que son distintos a la estimación de la edad, por ejemplo, los estudiantes de pregrado han desarrollado investigaciones en temas distintos a la estimación de la edad, asimismo, en la titulación de los alumnos en la especialidad de odontología forense de la Universidad Nacional Mayor de San Marcos y en otras universidades de la capital y del interior del país en los que he tenido la oportunidad de enseñar. Para lo sucesivo trataremos de investigar en distintos tópicos en torno a la identificación porque en la especialidad hay mucho por investigar.

¿Por qué no existe un número similar de investigaciones respecto a temas relacionados a responsabilidad profesional, consentimiento informado, historias clinicas o identificación?

Importante e interesante pregunta, ya que los temas que se mencionan son de suma importancia. Ahora más que nunca se necesitan tocar esos puntos, se están elaborando investigaciones referido a dichos temas, creo que todos los temas de investigación son importantes, sobre todo los que están relacionados con la supervisión de SUSALUD, SUNAFIL, siendo importante y necesario que el odontólogo conozca estos temas para no cometer mala praxis y evitar ser denunciado.

\section{¿Cómo ve usted el futuro de la odontología forense?}

Como especialista le veo un futuro excelente y creciendo a pasos agigantados, no nos olvidemos que San Marcos era la única universidad estatal que tenía la especialidad escolarizada y en la forma que venía funcionando era espléndida; además, a nivel de Sudamérica era el único país que tenía la especialidad escolarizada en una universidad estatal. No olvidemos que el Perú está muy adelantado en la especialidad de odontología forense a nivel de América, ello ha sido reconocido por otros países. Además, el suscrito, como profesor referente de la especialidad en América en los diversos certámenes científicos a nivel internacional, en los que también han participado docentes y alumnos de la especialidad, puedo decir que la odontología forense peruana ha sido muy elogiada y reconocida

Sin temor a equivocarme la odontología forense sigue siendo la especialidad del milenio y cada vez seremos más reconocidos a nivel internacional y nacional por la calidad de los odontólogos forenses egresados de la Universidad Nacional Mayor de San Marcos, la Decana de América.

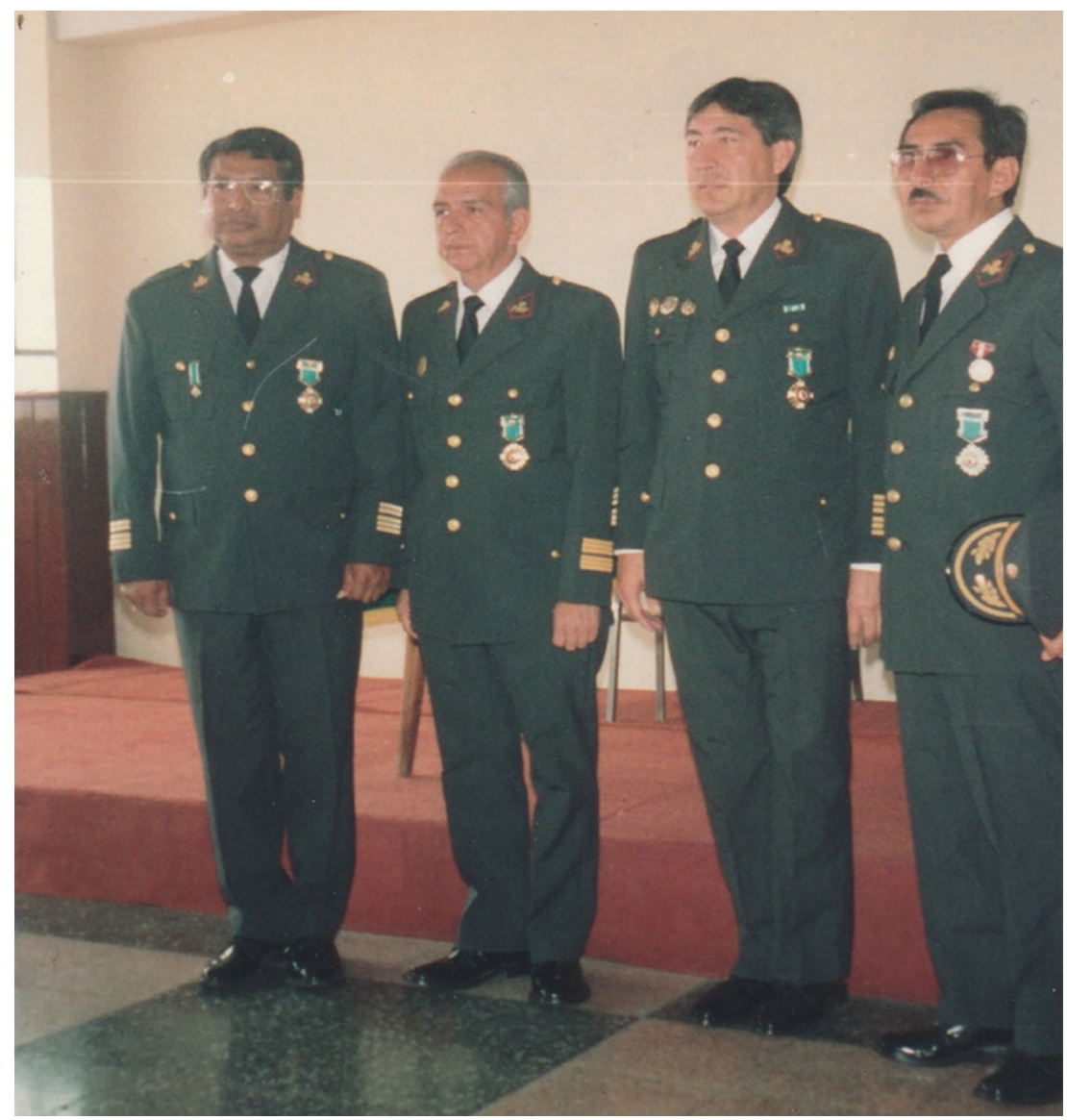

Profesor Hugo Caballero Cornejo se desempeñó como Coronel (S), Perito odontólogo y Jefe del Departamento Odontográfico de la Policía Nacional del Perú 


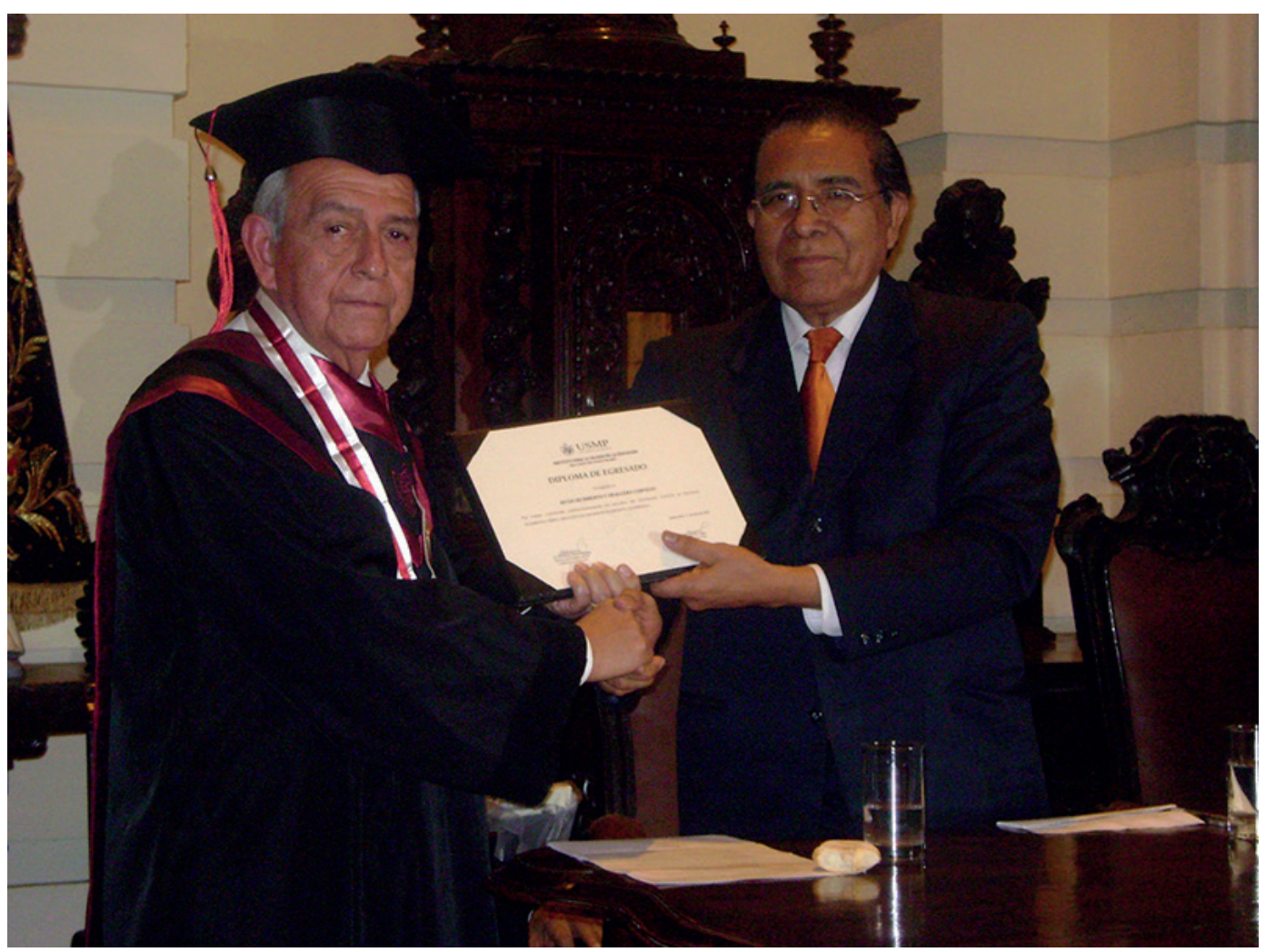

Ceremonia de graduación del doctorado

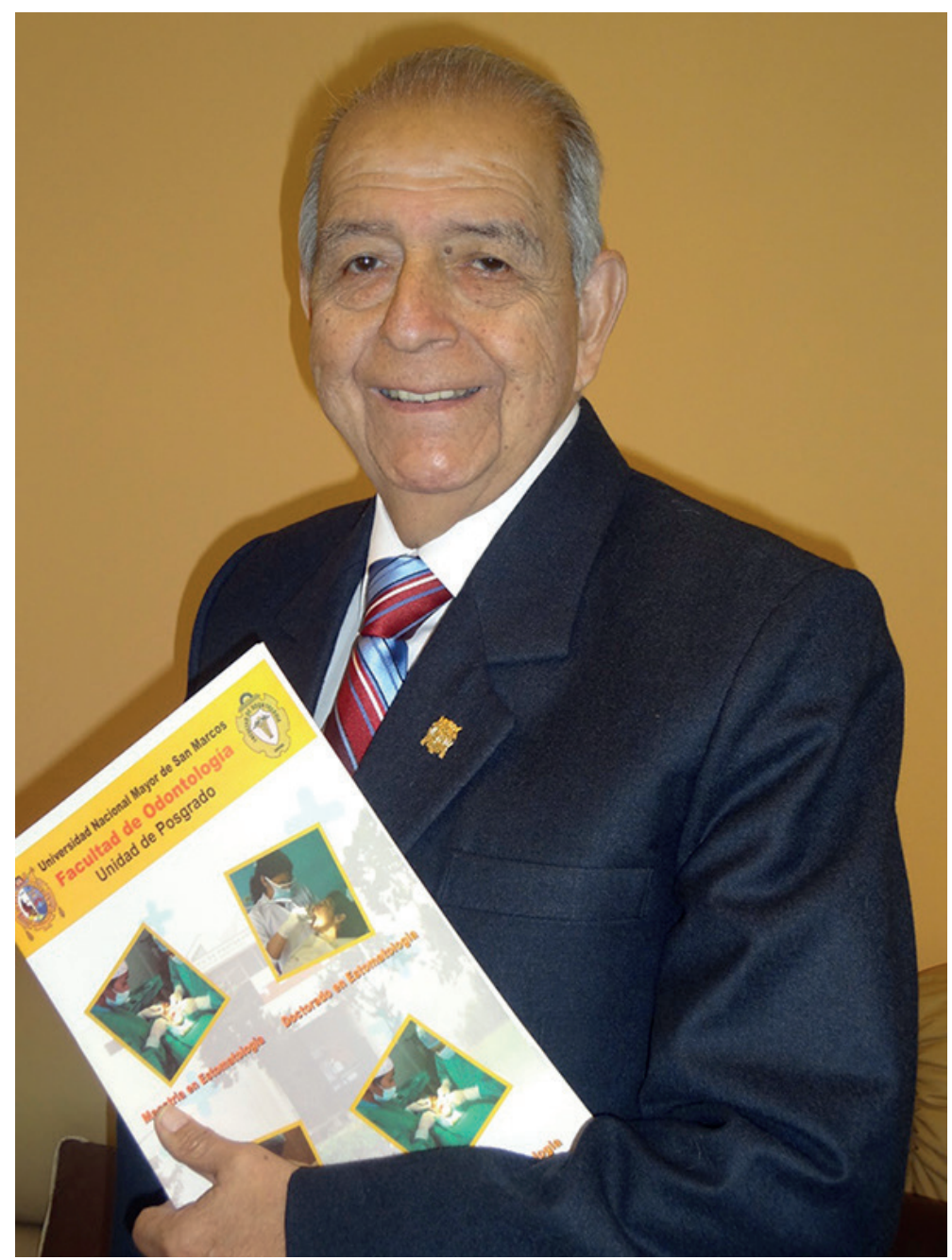

Fundador de la especialidad Odontología Forense en la Universidad Nacional Mayor de San Marcos 


\section{Referencias bibliográficas}

1. Caballero H. Odontología Legal y Forense. Lima: Editorial CEPREDIM - UNMSM; 2010.

2. Organización Internacional de Policía Criminal (INTERPOL). Guía para la Identificación de Víctimas de Catástrofes. 2009. [Consultado el 20 de junio del 2018]. Disponible en: https://www.interpol.int/News-and-media/Publications2/Guides-manuals2 
